\title{
Analysis of Microwave Passive Circuits Designed Using 3D Printing Techniques
}

\author{
Hector Garcia-Martinez ${ }^{1}$, Ernesto Avila-Navarro ${ }^{1}$, Alberto Rodriguez-Martinez ${ }^{2}$, \\ Miguel Angel de la Casa-Lillo ${ }^{3}$, German Torregrosa-Penalva ${ }^{2}$ \\ ${ }^{1}$ Materials Science, Optical and Electronic Technology, University Miguel Hernandez of Elche, \\ Avda. Universidad S/N, 03202, Elche, Alicante, Spain \\ ${ }^{2}$ Communication Engineering Department, University Miguel Hernandez of Elche, \\ Avda. Universidad S/N, 03202, Elche, Alicante, Spain \\ ${ }^{3}$ Bioengenieering Institute, University Miguel Hernandez of Elche, \\ Avda. Universidad S/N, 03202, Elche, Alicante, Spain \\ mhector@umh.es
}

\begin{abstract}
This work presents the results of a preliminary study conducted to assess the viability of using 3D printing techniques for the manufacturing of microwave passive circuits based on microstrip techniques, both for prototyping new lowcost devices and for fast and costless testing of new circuits designs. The study was aimed to test two crucial factors in the viability of these procedures: the performance of the circuit in terms of its functionality and the structural integrity of the resulting structure. A low-cost $3 \mathrm{D}$ printer is proposed for the dielectric substrate design of microstrip circuits. Standard PolyLactic Acid (PLA) material has been characterized in the microwaves frequencies for different substrate sizes and densities. To verify the proposed method, a low-pass stepped impedance filter was designed, simulated, and measured. Finally, a structural analysis using Non-Destructive Testing (NDT) ultrasonic techniques has been carried out based on deconvolution and resonant spectroscopy. Both, electronic and structural results, have shown the feasibility of using low-cost additive techniques in the design of microwave circuits.
\end{abstract}

Index Terms-3D printing; Additive manufacturing; Microwave circuits; Ultrasound NDT.

\section{INTRODUCTION}

Nowadays, 3D printers are widely used. Additive techniques are used as a quick and versatile solution in several fields, such as medicine, construction, robotics, and even as a viable solution for the manufacture of replace parts in space missions [1], [2].

Additive techniques are also being explored as a candidate for the manufacture of electronic devices. In this case, 3D printers' components are used as circuit support for different applications and areas, and even as part of the conductive elements of the circuits [3], [4]. The main problem is the lack of materials for 3D printers with good conductive characteristics, similar to those provided by copper and other conductive materials used in electronics. However, some studies have appeared in which sensors and other electronic devices are designed with 3D printers [5], [6].

Manuscript received 15 November, 2018; accepted 6 March, 2019.

This research was funded by a grant (Ref: DPI2016-80391-C3-2) from the Economy and Competitiveness Ministry of Spain.
In electronic devices design at $\mathrm{RF}$ and microwave frequencies, additive techniques are being used mainly in the design of waveguide devices and SIW (Substrate Integrated Waveguide). In this case, 3D printers are commonly used for the dielectric design and the metallization is done usually by chemical procedures [7], [8].

They can also be used in the design of the dielectric substrates in microstrip or stripline devices. Additive techniques offer some possibilities that are very interesting to design substrates with the dielectric properties (dielectric permittivity constant) necessary in each case depending on the design requirements. Some of the possibilities offered by this technology are the use of different densities in the material of the substrate, the use of multilayer devices, with several dielectric layers, each of them with the necessary height, and even the use of different materials in the substrate [9].

For the metallization of the different layers of the microstrip or stripline devices, the most appropriate method is to use copper sheets of very small thickness (typically 35 $\mu \mathrm{m})$ glued directly to the substrate layers, as it is done with conventional high-frequency laminates. Once the copper layer is adhered, the metal parts of the device can be milled. One of the goals of this work is to study the quality and performance of microwave circuits manufactured using this procedure.

Due to the inherent layered structure of the resulting 3D printed board, it is necessary to conduct an analysis to check whether the different layers of the board are properly fused, and the upper and lower copper sheets are properly glued. Errors in any of the previous, such as voids or bubbles in between the thermoplastic (PLA) layers or in the PLAEpoxy-copper interface, excess of epoxy, or lack of homogeneity in the density of the PLA layers, would compromise not only the structural integrity of the circuit, but its replicability and reliability also, and increase the electrical losses. To perform this analysis, we propose the use of ultrasound as it is fast, cheap, and very accurate, and can be used without damaging the circuits for their further use. The circuits will be evaluated using both deconvolution 
and spectroscopy [10], [11], applied to C-scans of the circuits to reveal any defect or structural problem.

\section{Description of the MANufacturing Process}

For the design and manufacturing of the dielectric substrate, as a part of the microstrip or stripline devices, a low-cost 3D printer (less than $500 €$ ) is used, in particular the model Prusa i3 Hephestos by BQ with a printing surface of $225 \times 210 \mathrm{~mm}^{2}$. Vertical and horizontal resolution is 60 $\mu \mathrm{m}$. The material deposition technique used is FDM (Fused Deposition Modelling) that allows the use of a variety of plastic materials, such as PLA, ABS (Acrylonitrile Butadiene Styrene) or Nylon. Additionally, a hot bed is used in the printer working area to control the temperature of the deposited material and improves the adhesion between the different layers of the substrate. Figure 1 shows the 3D printer used in this work.

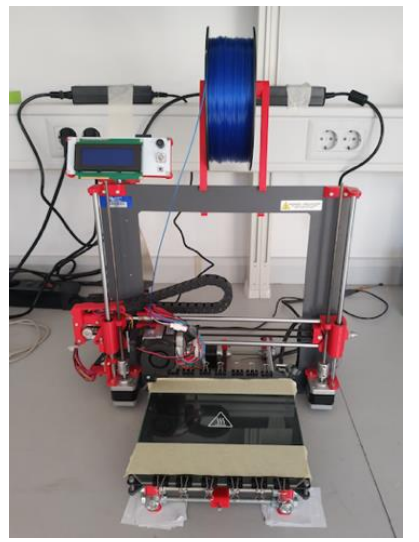

Fig. 1. 3D printer used in this paper - Prusa i3 Hephestos by BQ

The printer used has a numerical control based on a microcontroller that allows a medium degree of control in the design of the materials. By modifying the programming of the motor control and the extruder of the printer, a total control can be achieved in the quantity of material deposited in each area of the designed substrate, so the density of the material can be changed from one zone to another. In this way, it is possible to spatially modify the dielectric properties of the substrate and, therefore, the dielectric constant. This possibility of change in the dielectric constant will be used later to improve the characteristics of microwave circuits. Material densities ranging from $100 \%$ (maximum density allowed by the printer) to $15 \%$ have been tested.

The designed substrate has a structure in layers that can be defined in both, thickness and density. To give a greater rigidity in the substrate, all layers have a thickness of 100 $\mu \mathrm{m}$ and lower and upper part of the substrate have two layers of $100 \%$ density. The rest of the layers that form the substrate also have a thickness of $100 \mu \mathrm{m}$, but the density can be varied according to the needs of the design. Figure 2 shows a diagram with the different layers of the substrate.

The material used in the design of the dielectric substrate is standard low-cost PLA (less than $15 €$ per $1 \mathrm{Kg}$ roll), commonly used in 3D object design applications. The extrusion temperature of this material is $217 \mathrm{C}$ and the temperature used at the base of the hot bed is $48 \mathrm{C}$. For the metallization of the external layers of the substrate, a $35 \mu \mathrm{m}$ copper sheet is used directly glued to the plastic layers of the substrate with a non-conductive epoxy glue $(2216 \mathrm{~B} / \mathrm{A}$ Gray). For a correct adhesion of the metal sheet to the substrate, a uniform pressure is applied and it is cured at room temperature for 24 hours.

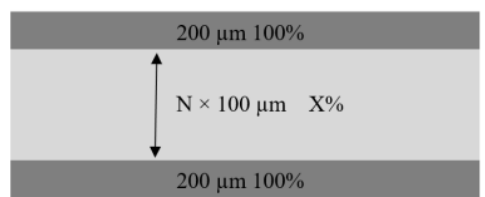

Fig. 2. Schematic of layers used in the 3D printing of the dielectric substrate.

Since the material used for the substrate design is not specific for high frequency applications, the characterization of the substrate (dielectric constant and loss tangent) at microwave frequencies is needed before the design of the circuits. The method used to calculate these two parameters is a mixed method of simulation and measurement based on a quarter-wave resonator and a transmission line, both in microstrip technology. The electrical properties of the material are modified in the simulation until the results match with the measured ones. For circuits simulation, EMPro software by Keysight was used. For the measurement of the Scattering parameters, a Vector Network Analyzer was used (E8363B by Agilent Technologies). Figure 3 shows one of the circuits designed to obtain the characteristic parameters of the substrate (density $100 \%$ ) and a table with the calculated dielectric constant for each density of material evaluated. It is shown that varying the density it is possible to design the relative permittivity of the substrate. The loss tangent obtained remains practically constant around 0.02 . It is a high value, but similar to FR4 substrate, widely used in the design of microwave devices below $5 \mathrm{GHz}$. It should be noted that different heights of substrate have been evaluated (from 1.6 $\mathrm{mm}$ to $0.4 \mathrm{~mm}$ ) obtaining the same results as it is shown in Table I, in all cases.

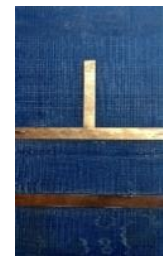

Fig. 3. Microstrip circuit used in the characterization of the $100 \%$ density substrate.

TABLE I. RESULTS FOR THE DIFFERENT DENSITIES EVALUATED.

\begin{tabular}{|c|c|}
\hline Percentage of Material & Relative Permittivity \\
\hline $100 \%$ & 2.88 \\
\hline $90 \%$ & 2.78 \\
\hline $70 \%$ & 2.56 \\
\hline $50 \%$ & 2.31 \\
\hline $30 \%$ & 2.1 \\
\hline $15 \%$ & 1.86 \\
\hline
\end{tabular}

\section{STEPPED IMPEDANCE LOW-PASS FILTER WITH AdDITIVE TECHNIQUES}

To test the design possibilities of microstrip circuits at microwave frequencies, manufacturing the substrate using additive techniques, we propose the design of a filter in microstrip technology. It is a 5-th order stepped impedance Butterworth low-pass filter with $2 \mathrm{GHz}$ cut-off frequency, 
implemented with high and low impedance microstrip line sections that emulate the inductor and capacitor behaviour, respectively.

Dielectric substrate has been manufactured with standard PLA material, $1.6 \mathrm{~mm}$ height and $100 \%$ density in all layers. Dielectric constant is extracted from the results shown in Table I: $\varepsilon_{\mathrm{r}}=2.88$.

To reduce errors in the milling procedure of the transmission lines, a width of $0.75 \mathrm{~mm}$ has been imposed as a limit for the high impedance lines (110.2 $\Omega$ in the designed substrate), while for the low impedance lines it has been used a width of $10 \mathrm{~mm}(24.4 \Omega)$. Two $50 \Omega$ line sections of $3.86 \mathrm{~mm}$ wide have been included in the input and output ports of the filter.

Lengths of the different sections of high and low impedance are obtained by applying the following relationships:

$$
\left\{\begin{array}{l}
\beta L_{H}=\frac{L Z_{0}}{Z_{H}}, \\
\beta L_{L}=\frac{C Z_{L}}{Z_{0}},
\end{array}\right.
$$

where $\mathrm{L}$ and $\mathrm{C}$ are the normalized inductors and capacitors of the Butterworth design table, $\mathrm{Z}_{\mathrm{H}}$ and $\mathrm{Z}_{\mathrm{L}}$ are the impedances for the high and low impedance lines (110.2 $\Omega$ and $24.4 \Omega), Z_{0}$ is the characteristic impedance of the filter $(50 \Omega)$ and $\beta=2 \pi / \lambda$. Lengths of each sections are shown in Table II.

Figure 4 shows the manufactured filter, where the different line sections of the filter can be seen. Two $50 \Omega$ SMA connectors have been added and a conductive epoxy has been used to improve the electrical contact between connectors and the circuit.

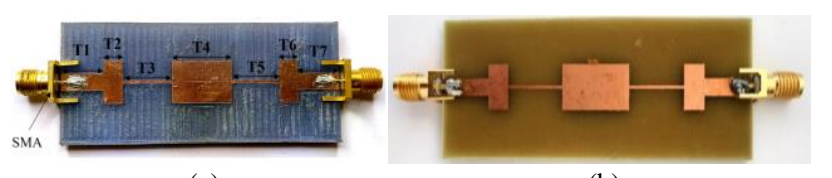

(a)

(b)

Fig. 4. Stepped impedance low-pass filter prototype: (a) 3D printer; (b) commercial FR4.

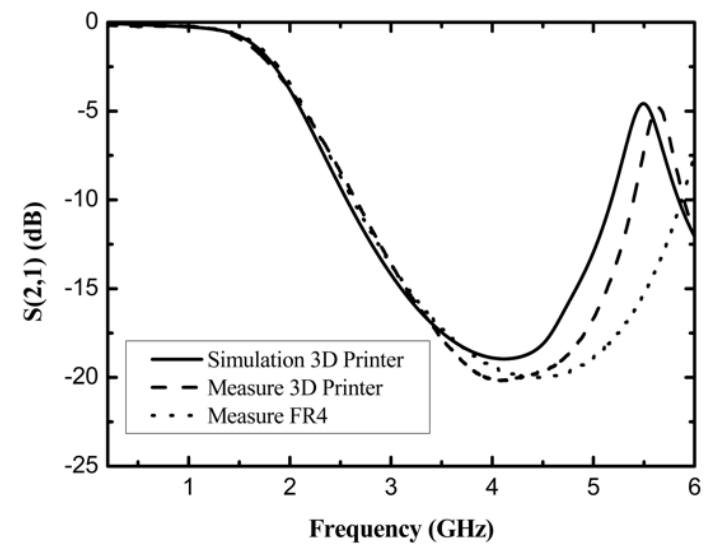

Fig. 5. Measured and simulated results for the designed low-pass filter.

TABLE II. LENGTH OF MICROSTRIP LINES.
\begin{tabular}{|c|c|c|c|}
\hline Line & Length $(\mathbf{m m})$ & Line & Length $(\mathbf{m m})$ \\
\hline $\mathrm{T} 1, \mathrm{~T} 7$ & $3.86 \mathrm{~mm}$ & T4 & $14.67 \mathrm{~mm}$ \\
\hline $\mathrm{T} 2$ & $4.63 \mathrm{~mm}$ & $\mathrm{~T} 5$ & $11.66 \mathrm{~mm}$ \\
\hline $\mathrm{T} 3$ & $11.66 \mathrm{~mm}$ & $\mathrm{~T} 6$ & $4.63 \mathrm{~mm}$ \\
\hline
\end{tabular}

Figure 5 shows the simulated and measured transfer function of the filter. As can be seen, very good results are obtained in the whole frequency band analysed, obtaining a cut-off frequency of $1.95 \mathrm{GHz}$, very close to the designed 2 GHz. FR4 results are included for comparison.

\section{Structural Analysis}

As stated in the introduction, the structural analysis has been conducted using ultrasonic non-destructive testing techniques. The circuit samples were scanned in an immersion basin in distilled water, using a $5 \mathrm{MHz}$ focused transducer from OLYMPUS as pulse-echo transducer. The XYZ scanner performed a C-scan taking A-scans every 200 $\mu \mathrm{m}$ in all the surface of the circuits, using the non-stripped flat homogeneous copper side as incidence surface. The acquisition equipment was an SE-TX06-00 from KTU electronics with sampling rate $100 \mathrm{MHz}$.

The resulting A-scans were processed using two different techniques. Before processing, an automatic deconvolution was performed as described in [10], using the averaged front-echo pulse as reference for the stripping of the successive layers of the signals. The first Time-of-Flight matrix is used to align all the A-scans to the front surface of the un-stripped copper layer, and then the absolute value of the envelope is calculated of the whole $\mathrm{C}$-scan to produce magnitude 2D images that can be used to explore the inner layers of the material. Finally, a spectroscopy analysis was performed as described in [11], using, again. the averaged front-echo pulse as reference. This processing produces a 3D matrix with the resonant spectrum, which can be used to find singularities in the inner structure of the material. We encourage readers, interested in these techniques, to review the provided references.

In order to have a visual reference and to make more clear the information provided by this analysis, we show the results of the analysis performed on the circuit shown in Fig. 4(a) compared to that made to a circuit with the same design but produced using the classic commercial two-layered FR4 circuit board with similar dimensions (1.5 mm thickness), which can be seen in Fig. 4(b). Note the homogeneity of the composite surface compared to that seen in Fig. 4(a) for the printed substrate. Next figures show examples of the results obtained with the ultrasonic analysis.

Figure 6 shows the resulting 2D envelope image with time of flight adjusted just a few microns before the back surface of the board.

As can be seen in the case of the PLA based circuit (Fig. 6(a), there are some irregularities in the interface between the copper layer and the back face of the PLA. These irregularities are due to the irregular distribution of the epoxy used as glue and its corresponding changes in density during the curing process.

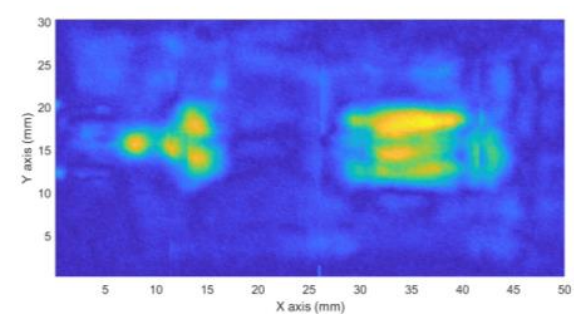

(a) 


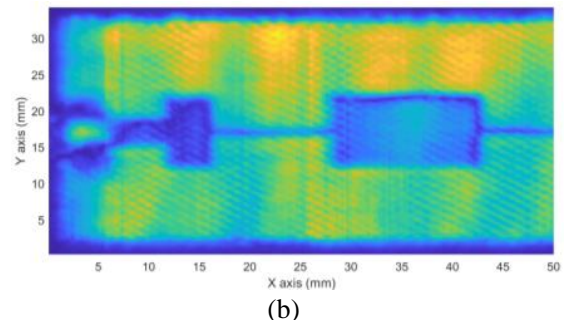

(b)

Fig. 6. Magnitude distribution: (a) 3D printed; (b) FR4.

On the other hand, for the FR4 based circuit, the interface between materials can be clearly seen, where the latticed structure of the latest layers of the fiber matrix that form the composite FR4 can be distinguished.

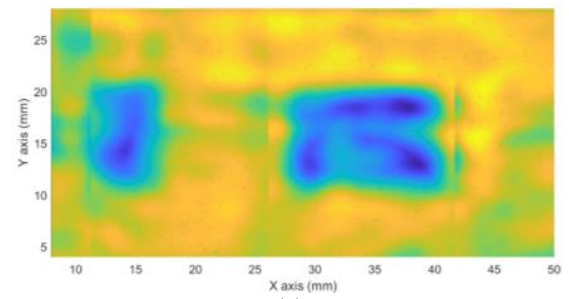

(a)

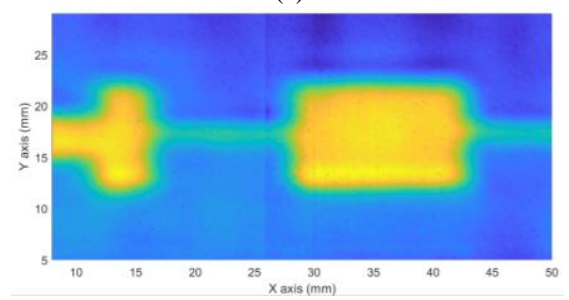

(b)

Fig. 7. Spectroscopy at the back-face layer: (a) 3D printed; (b) FR4.

Spectroscopy can be used to overcome the problems of amplitude masking, and to gain resolution in terms of depth also. In this particular case, the transfer function between the received signal and the reference signal can be used for the analysis.

Figure 7 shows the results of the spectroscopy of the same depth, where now only the interface copper-substrate of the back surface is shown, and where, again, we can see the inhomogeneous distribution of the epoxy if we compare the 3D printed circuit (Fig. 7(a)) with the FR4 circuit (Fig. 7(b)).

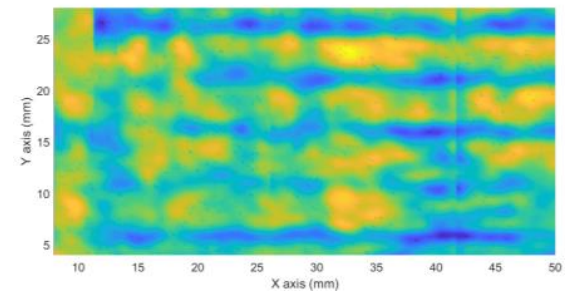

(a)

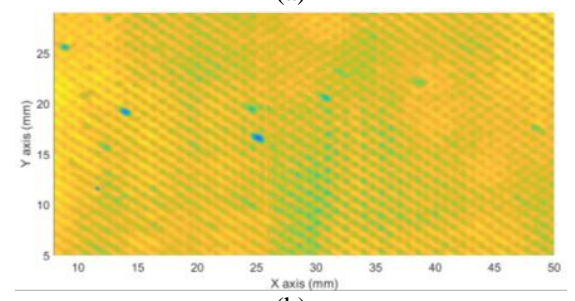

(b)

Fig. 8. Spectroscopy after front-face layer: (a) 3D printed; (b) FR4.

Finally, figure 8 shows a spectroscopy image of the material just a few microns $(\sim 0.1 \mathrm{~mm})$ after the front facecopper sheet. The inner structure can be clearly seen in both cases now, as horizontal thick layers in case of the 3D printed circuit (Fig. 8(a)) and the fibre matrix of the FR4 circuits (Fig. 8(b)).

\section{Conclusions}

In this work, the use of additive techniques based on lowcost $3 \mathrm{D}$ printers and standard plastic material for the design of passive microwave devices has been validated. PLA material used has been characterized and the manufacturing method has been tested with a typical microstrip filter in microwaves applications. The proposed circuit has been analysed both electronically and structurally, offering good results. The ultrasonic analysis has shown only minor gluing maladjustments, which can be refined in further test. However, there are no voids and the layers are properly fused. The importance of the process of bonding the layers of the substrate and the copper sheets to avoid irregularities has been confirmed. The proposed manufacturing method allows to include the design of the dielectric substrate as a parameter in the design of microstrip circuits.

\section{REFERENCES}

[1] W. Gao, Y. Zhang, D. Ramanujan, K. Ramani, Y. Chenc, Ch. B. Williams, Ch. C. L. Wange, Y. C. .Shin, S. Zhang, P. D. Zavattieri, "The status, challenges, and future of additive manufacturing in engineering", Computer-Aided Design, vol. 69, pp. 65-89, 2015 DOI: 10.1016/j.cad.2015.04.001.

[2] NASA, "3D Printing in Zero-G Technology Demonstration," 2015 [Online]. Available: https://www.nasa.gov/mission_pages/station/ research/experiments /1115.html,.

[3] E. Macdonald, R. Salas, D. Espalin, M. Perez, E. Aguilera, D. Muse, R. B. Wicker, "3D Printing for the Rapid Prototyping of Structural Electronics", IEEE Access, vol. 2, pp. 234-242, 2014. DOI: 10.1109/ACCESS.2014.2311810.

[4] C. Kim, D. Espalin, M. Liang ; H. Xin, A. Cuaron, I. Varela, E. Macdonald, R. B. Wicker, "3D Printed Electronics With High Performance, Multi-Layered Electrical Interconnect", IEEE Access, vol. 5, pp. 25286-25294, 2017. DOI 10.1109/ACCESS.2017.2773571.

[5] P. Flowers, Ch. Reyes, Sh. Ye, M. J. Kim, B. J. Wiley, "3D printing electronic components and circuits with conductive thermoplastic filament", Additive Manufacturing, vol.18, pp. 156-163, 2017. DOI: 10.1016/j.addma.2017.10.002.

[6] M. Saari, B. Xia, B. Cox, P. S. Krueger, "Fabrication and analysis of a composites 3D printed capacitive force sensor", 3D Printing and Additive Manufacturing, vol. 3, pp. 136-141, 2016. DOI: $10.1089 / 3 \mathrm{dp} .2016 .0021$

[7] G. Addamo, O. A. Peverini, D. Manfredi, F. Calignano, F. Paonessa, G. Virone, R. Tascone, G. Dassano, "Additive Manufacturing of KaBand Dual-Polarization Waveguide Components", IEEE Transactions on Microwave Theory and Techniques, vol. 66, pp. 3589-3596, 2018. DOI: 10.1109/TMTT.2018.2854187

[8] E. Massoni, L. Silvestri, G. Alaimo, S. Marconi, M. Bozzi, L. Perregrini, F. Auricchio, "3-D Printed Substrate Integrated Slab Waveguide for Single-Mode Bandwidth Enhancement", IEEE Microwave and Wireless Components Letters, vol. 27, no. 6, pp. 536538, 2017. DOI: 10.1109/LMWC.2017.2701323.

[9] J. A. Byford, M. I. M. Ghazali, S. Karuppuswami, B. L. Wright, P. Chahal, "Demonstration of RF and Microwave Passive Circuits Through 3-D Printing and Selective Metallization", IEEE Transactions on Components, Packaging and Manufacturing Technology, vol. 7, no. 3, pp. 463-471, 2017. DOI: 10.1109/TCPMT.2017.2651645.

[10] A. Rodríguez, L. Svilainis, V. Dumbrava, A. Chaziachmetovas, A Salazar, "Automatic simultaneous measurement of phase velocity and thickness in composite plates using iterative deconvolution", $N D T \& E$ Int., vol. 66, pp. 117-127, 2014. DOI: 10.1016/j.ndteint.2014.06.001.

[11] T. E. Gómez Alvarez-Arenas, D. A. Soto, "Characterization of mineral paper by air-coupled ultrasonic spectroscopy", Ultrasonics, vol. 52, no. 6, pp. 794-801, 2012. DOI: 10.1016/j.ultras.2012.02.012. 